\title{
MPTP-induced model of Parkinson's disease in heat shock protein 70.1 knockout mice
}

\author{
HYUN KYUNG PARK ${ }^{1}$, AH RANG CHO ${ }^{2}$, SEUNG CHUL LEE $^{3}$ and JU YEON BAN ${ }^{4}$ \\ Departments of ${ }^{1}$ Emergency Medicine, and ${ }^{2}$ Psychiatry, School of Medicine, Kyung Hee University, Seoul; \\ ${ }^{3}$ Department of Emergency Medicine, Dongguk University Ilsan Hospital, Seoul; ${ }^{4}$ Department of Pharmacology, \\ College of Dentistry and Institute of Tissue Regeneration Engineering, Dankook University, Cheonan, Republic of Korea
}

Received December 20, 2011; Accepted March 12, 2012

DOI: $10.3892 / \mathrm{mmr} .2012 .839$

\begin{abstract}
Heat shock proteins (HSPs), molecular chaperones that assist in protein folding, have become a research focus in Parkinson's disease (PD) since the pathogenesis of PD is characterized by intracellular protein misfolding and inclusion body formation. This study investigated the effect of the knockout (KO) of the Hsp70.1 (approved gene symbol Hspalb) gene on the sensitivity of murine nigrostriatal dopaminergic neurons to 1-methyl-4-phenyl-1,2,3,6-tetrahydropyridine (MPTP)-induced toxicity. We confirmed changes in motor coordination and tyrosine hydroxylase (TH) immunoreactivity in the substantia nigra following MPTP treatment of C57BL/6 normal mice and Hspalb KO mice. MPTP treatment led to motor control impairment and induced TH-positive dopaminergic neurodegeneration in normal mice. Compared to untreated normal mice, rotarod duration and the density of TH-positive neurons in the substantia nigra were also significantly lower in untreated KO mice $(\mathrm{p}<0.01)$. MPTPtreated $\mathrm{KO}$ mice had markedly decreased rotarod duration and reduced density of TH-positive neurons, compared to MPTPtreated normal mice. These results indicate that Hspalb KO mice are more vulnerable to the neurotoxic effects of MPTP and are consistent with the hypothesis that HSPs represent an important molecular target for neuroprotective strategies in the treatment of PD.
\end{abstract}

\section{Introduction}

Parkinson's disease (PD) is a neurodegenerative disease characterized by progressive deterioration of motor function due to selective loss of dopaminergic neurons in the nigrostriatal pathway. PD is clinically characterized by tremor, rigidity, postural abnormalities and bradykinesia. Although

Correspondence to: Professor $\mathrm{Ju}$ Yeon Ban, Department of Pharmacology, College of Dentistry, Dankook University, Anseodong, Dongnam-gu, Cheonan 330-714, Republic of Korea

E-mail: jyban@dankook.ac.kr

Key words: Hspa1b, heat shock protein 70, MPTP, Parkinson's disease the etiopathogenesis of the processes underlying clinical deterioration remains unknown, several potential key factors, including genetic background and endogenous or environmentderived neurotoxins, have been proposed (1). Several genetic mutations have been identified, including $\alpha$-synuclein, parkin, UCHL1, DJ-1, PINK1 and LRRK2 (2), and provide clues to the molecular pathways involved in the vulnerability and loss of dopaminergic cells during disease progression. Multiple mechanisms are thought to be involved in cell death in PD. These include abnormal protein interactions in the ubiquitinproteasome system (UPS), oxidative stress, mitochondrial dysfunction, apoptosis, inflammation and excitotoxicity (3), although the precise roles of these remain unclear.

Heat shock proteins (HSPs) have a protective role against a variety of adverse conditions, including oxidative stress (4). The protection of cells by HSPs is attributed to their molecular chaperone function, which facilitates nascent protein folding, refolding, or degradation of abnormally folded protein $(5,6)$. Given the critical role of HSPs in protein stabilization, folding, and assembly, it is not surprising that HSPs are involved in the pathogenesis of a variety of neurodegenerative disorders characterized by abnormal protein aggregation. Evidence indicates that HSPs may be involved in neuronal cell death in PD, a disease characterized by conformational changes in the proteins that result in misfolding, aggregation and intracellular Lewy body formation (7-9). The overexpression of Hsp70 has been shown to prevent dopaminergic neuronal loss associated with $\alpha$-synuclein in Drosophila (9). Hsp70 gene transfer to the dopamine neurons by a recombinant adeno-associated virus also protected the mouse dopaminergic system against 1-methyl-4-phenyl-1,2,3,6tetrahydropyridine (MPTP)-induced loss of dopaminergic neurons (10). Moreover, the induction of Hsp70 expression by prior exposure to heat shock offers some protection against 1-methyl, 4-phenyl, pyridinium ion (MPP)-induced neuronal cell death in cultured PC12 cells (11). In addition, a recent epidemiological study reported that susceptibility to PD may be associated with a potential polymorphism in the $5^{\prime}$ promoter region of the HSP70.1 gene (12). Therefore, HSPs may represent an important molecular target for neuroprotective strategies in PD treatments.

HSPs are classified into discrete families based on their molecular mass: Hsp100, Hsp90, Hsp70, Hsp60, Hsp40, 
and small Hsp families (13). One of the most conserved is the Hsp70 family (14). Three genes encoding members of the Hsp70 family have been defined: Hsp70.1 (Hspa1b), Hsp70.2 (Hspa2), and Hsp70.hom (Hspa1l) (15). Hspalb and Hspa2 encode an identical protein, the major heat-inducible Hsp70. Hspalb is also expressed constitutively at low levels. Hspall encodes a protein that shares $90 \%$ amino acid sequence identity with Hsp70 but is not heat-inducible.

In order to understand the role of Hspalb in the pathogenesis of PD, this study investigated the effect of the knockout (KO) of the Hspalb gene on MPTP-induced toxicity. We compared changes in motor function using rotarod test and the density of tyrosine hydroxylase (TH)-immunoreactive neurons after MPTP treatment in C57BL/6 normal mice and Hspalb KO mice.

\section{Materials and methods}

Animals and MPTP administration. All experiments were carried out in accordance with the animal care guidelines of the National Institutes of Health and Animal Care Committee of the Medical College, Kyung Hee University. Male C57BL/6 mice (8 weeks old, weighing 25-30 g) were purchased from SLC Inc. (Hamamatsu, Shizuoka, Japan) and Hspalb KO mice were purchased from Macrogen Inc. (Seoul, Korea). The mice were housed at standard temperature $\left(22 \pm 2^{\circ} \mathrm{C}\right)$ on a 12 -h light/dark cycle, with free access to food and water. The C57BL/6 normal and Hspalb KO mice were each divided randomly into two subgroups: normal controls, MPTP-treated normal controls, $\mathrm{KO}$ mice, and MPTP-treated KO mice. To generate the PD model, mice were injected intraperitoneally with MPTP-HCl (Sigma, St. Louis, MO, USA) in saline at a dose of $30 \mathrm{mg} / \mathrm{kg}$ at 24-h intervals on 5 consecutive days. The control group was injected with saline on the same schedule.

Rotarod test. An accelerating rotarod (MED Associates Inc., St. Albans, VT, USA) was used to measure motor balance and coordination in mice. Mice were required to maintain their balance on a horizontal rotating rod (diameter, $3 \mathrm{~cm}$ ) and rotation speed was increased every $30 \mathrm{sec}$ by $4 \mathrm{rpm}$. Five mice were tested at the same time, separated by large disks. A trial started when the mouse was placed on the rotating rod, and it stopped when the mouse fell down or when 4 min had passed. Falling down activated a switch that automatically stopped a timer. At $24 \mathrm{~h}$ after the last MPTP or saline injection, the mice were pretrained three times with $1 \mathrm{~h}$ intervals with an accelerating rod speed mode. Then, the time they remained on the rod was recorded with a maximum of $240 \mathrm{sec}$ for successive rotational speeds.

Tyrosine hydroxylase (TH) immunohistochemistry. Animals were sacrificed by chloral hydrate overdose $(300 \mathrm{mg} / \mathrm{kg})$ $24 \mathrm{~h}$ after the last MPTP or saline injection, and were then perfused transcardially with $4 \%$ paraformaldehyde in $0.05 \mathrm{M}$ phosphate buffer (PB). The brains were removed, postfixed and cryoprotected. Immunohistochemistry was performed as described in a previous study (16) on free-floating cryomicrotome-cut sections ( $40 \mu \mathrm{m}$ thick) that encompassed the entire substantia nigra. After incubation with $3 \% \mathrm{H}_{2} \mathrm{O}_{2}$ in $0.05 \mathrm{M}$ PBS, the sections were stained overnight at room temperature

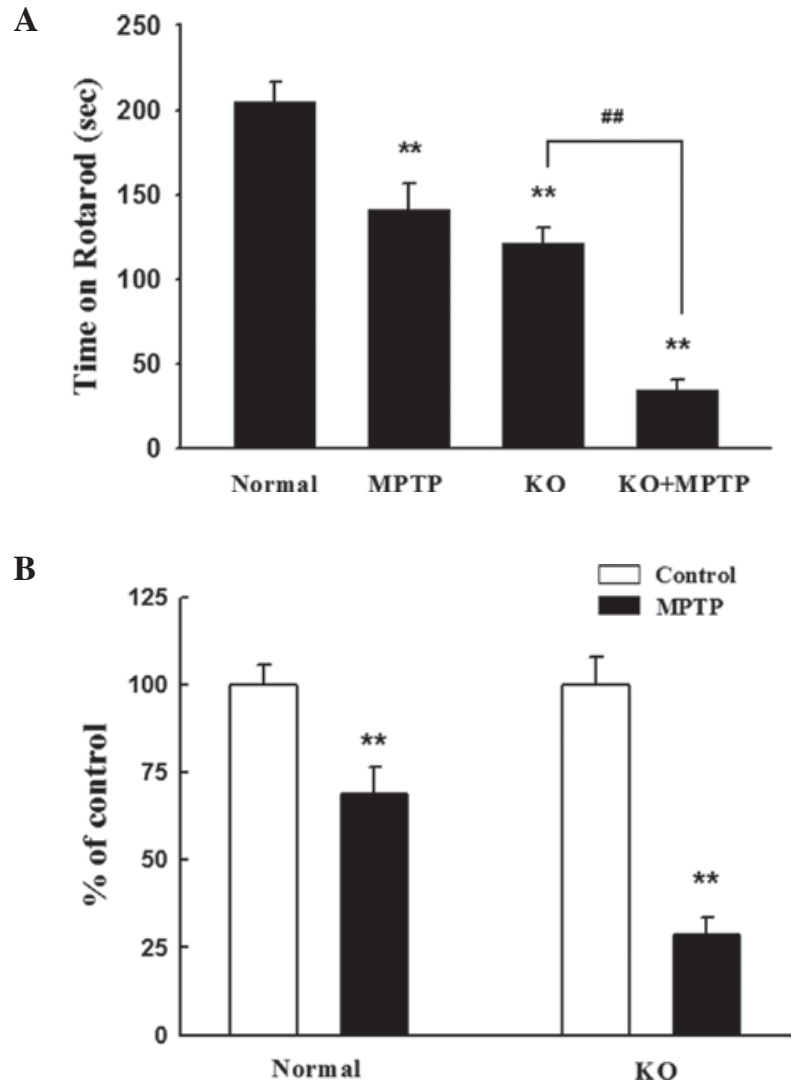

Figure 1. Effect of a knockout (KO) of Hspalb on MPTP-induced motor function impairment. (A) Motor performances on rotarod of normal and Hspalb $\mathrm{KO}$ mice in the presence or absence of MPTP. The data are expressed as the mean \pm SEM. ${ }^{* *} \mathrm{p}<0.01$, compared to the untreated normal group; ${ }^{\# \#} \mathrm{p}<0.01$, compared to the untreated KO group. (B) The rotarod duration of untreated groups was regarded as $100 \%$. The data are expressed as the mean \pm SEM. ${ }^{* *} \mathrm{p}<0.01$, compared to the untreated group.

using an anti-TH (1:1000; Santa Cruz Biotechnology, Santa Cruz, CA, USA) primary antibody for dopamine neurons. The tissue sections were incubated with biotinylated antirabbit IgG (Vector Laboratories Inc., Burlingame, CA, USA) for $1 \mathrm{~h}$ at room temperature, incubated with $A B C$ reagent (Vector Laboratories Inc., Burlingame, CA, USA) for $1 \mathrm{~h}$ at room temperature, and incubated for $2 \mathrm{~min}$ in $0.02 \%$ diaminobenzidine and $0.003 \% \mathrm{H}_{2} \mathrm{O}_{2}$ in $1 \mathrm{M}$ Tris-buffered saline ( $\mathrm{pH}$ 7.5). After the reaction, the tissue sections were mounted on gelatin-coated slides, dried, dehydrated and covered.

Statistical analysis. Values are expressed as mean \pm SEM. The significance of differences between groups was statistically analyzed using one-way analysis of variance (ANOVA), followed by Tukey's test. A value of $p<0.05$ was considered to indicate statistical significance.

\section{Results}

Effect of the knockout of Hspalb on MPTP-induced motor impairment. To evaluate MPTP-induced impairment of motor coordination, we used the rotarod test, which assessed the ability of mice to maintain their balance on a rotating cylinder. MPTP treatment of normal mice led to motor impairment (Fig. 1A). Time on the rotarod was significantly decreased 
A
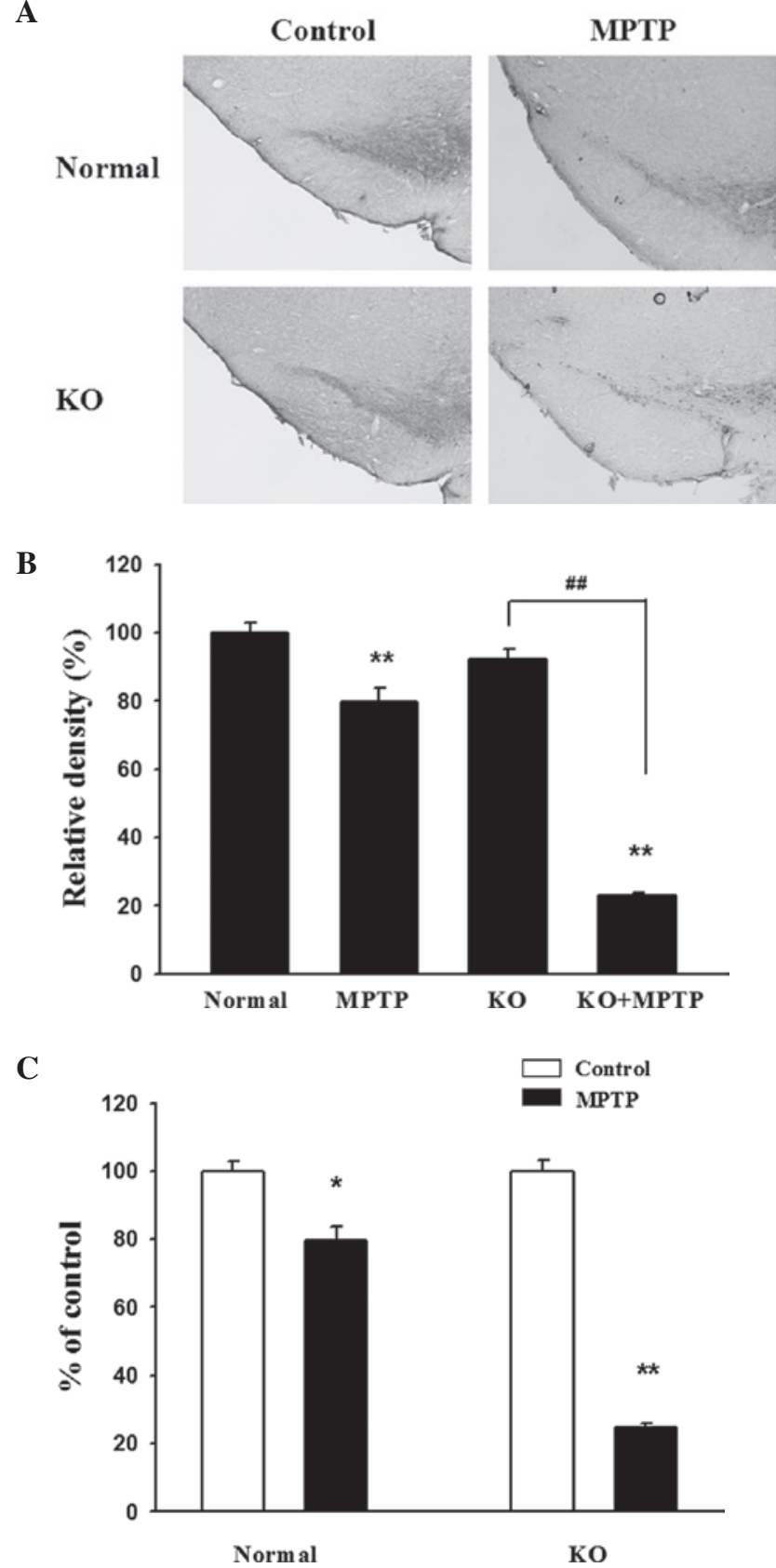

Figure 2. Effect of a knockout (KO) of Hspalb on MPTP-induced tyrosine hydroxylase (TH) immunoreactive neurodegeneration. (A) Representative photomicrographs showing TH-positive dopaminergic neurons in the substantia nigra of each group. (B) The density of TH-positive dopaminergic neurons in the substantia nigra. The data are expressed as the mean \pm SEM ${ }^{* *} \mathrm{p}<0.01$, compared to the untreated normal group; ${ }^{* \#} \mathrm{p}<0.01$, compared to the untreated $\mathrm{KO}$ group. (C) The density of TH-positive dopaminergic neurons of the untreated groups was regarded as $100 \%$. The data are expressed as the mean \pm SEM. $" \mathrm{p}<0.05$ compared to the untreated group; ${ }^{* *} \mathrm{p}<0.01$ compared to the untreated group.

to $141.40 \pm 15.59 \mathrm{sec}$ in the MPTP-treated normal group, compared with $205.00 \pm 11.76 \mathrm{sec}$ in the untreated normal group $(\mathrm{p}<0.01)$. Interestingly, in the absence of MPTP treatment, Hspalb KO mice showed motor deficits. MPTP-treated $\mathrm{KO}$ mice significantly showed motor impairment compared to untreated $\mathrm{KO}$ mice.

To investigate whether mice with an Hspalb deficiency are more vulnerable to MPTP-induced toxicity, we compared changes in motor coordination due to MPTP treatment in the untreated groups. As shown in Fig. 1B, the MPTP-treated KO group showed marked impairment of motor coordination compared to the MPTP-treated normal group. Motor impairment in the MPTP-treated KO group decreased by approximately $72 \%$ compared to the untreated $\mathrm{KO}$ group, while the MPTP-treated normal group decreased by approximately $32 \%$ compared to the untreated normal group, indicating that Hspalb $\mathrm{KO}$ mice are more vulnerable to MPTP-induced toxicity.

TH immunohistochemistry. Fig. 2A shows the TH immunoreactivity of dopaminergic neurons in the substantia nigra sections of normal and Hspalb KO mice after treatment with MPTP. We confirmed a significant reduction in the density of TH-positive dopaminergic neurons in the substantia nigra of MPTP-treated normal mice, compared to untreated normal mice (Fig. 2B). The MPTP-treated KO group also had significantly fewer $\mathrm{TH}$-positive neurons in the substantia nigra than the untreated KO group $(\mathrm{p}<0.01)$. As shown in Fig. 2C, the MPTP-treated KO group showed a marked reduction of TH-positive neurons compared to the MPTP-treated normal group. The density of TH-positive neurons in the MPTPtreated KO group decreased by approximately $75 \%$ compared to the untreated KO group, while the MPTP-treated normal group decreased by approximately $20 \%$ compared to the untreated normal group, also indicating that Hspalb KO mice are more susceptible to MPTP-induced toxicity.

\section{Discussion}

The neurotoxin MPTP is capable of producing biochemical and neuropathological defects similar to those observed in PD patients(17),including progressiveloss of dopaminergic neurons in the substantia nigra and decreases in striatal dopamine levels. Therefore, it is widely used as an experimental model of PD (18). The neurotoxicity of MPTP depends on the transformation of MPTP to its bioactive form $\mathrm{MPP}^{+}$, which is taken up into dopaminergic (DA) neurons by their DA transporters. $\mathrm{MPP}^{+}$ inhibits mitochondrial complex I, leading to decreased ATP production, increased generation of reactive oxygen species, apoptosis, and endoplasmic reticulum stress due to accumulation of unfolded proteins $(10,19,20)$.

Molecular chaperones, such as HSP70s, not only play crucial roles in protein folding and degradation, but also contribute to antioxidant defenses and anti-apoptotic pathways, which have been clearly implicated in the pathogenesis of PD (21). Several studies have reported that the Hsp70 gene protects against PD. Genetic studies in Drosophila showed that the overexpression of Hsp70 suppressed polyglutamine-induced neurodegeneration in vivo (22). Shen et al reported that the induction of Hsp70 is critical to the neuroprotection afforded by geldanamycin against MPTP-induced neurotoxicity in the mouse brain (23). Moreover, induction of Hsp70 by prior exposure to heat shock protects against $\mathrm{MPP}^{+}$-induced neuronal cell death in cultured PC12 cells (11) and against $\alpha$-synuclein aggregation in human $\mathrm{H} 4$ neuroglioma cells $(7,8)$. These results highlight the possibility of using Hsp70 as a potential therapy for PD. Nagel et al also reported that Tat-Hsp70 prevented the MPTP-mediated loss of dopaminergic midbrain neurons in culture as well as 
in the substantia nigra in a mouse model of PD (24). However, some reports have questioned the therapeutic potential of heat shock proteins in PD. Gao et al reported that ubiquitous transgenic overexpression of Hsp70 did not suppress MPTP-induced nigrostriatal dopaminergic neuronal damage at either the soma or the axon terminals of dopaminergic neurons (25). Moreover, Hsp70 overexpression did not favor the recovery of striatal axon terminals after MPTP treatment. Although HSP70 has been found to reduce $\alpha$-synuclein aggregation and toxicity in mice (7), that finding was not repeated in a more recent study (26). These results indicate that Hsp70 alone is not sufficient to reduce MPTP-induced dopaminergic neuronal damage in mice. Therefore, it remains unclear whether Hsp70 overexpression or activation may be used as a therapeutic approach in PD patients and these effects require further study.

With ageing, the level of HSPs is decreased and they are insufficient to maintain homeostasis of cellular proteins, which may give rise to certain diseases (27). In this study, we investigated whether mice with Hsp70 deficiency are more vulnerable to MPTP-induced toxicity in an animal model of PD that used Hspalb KO mice. Our results show that MPTP-treated Hspalb $\mathrm{KO}$ mice have more severe motor impairment compared to MPTP-treated normal mice. TH-immunoreactive dopaminergic neurons were also fewer in MPTP-treated Hspalb KO mice than in MPTP-treated normal mice. These results indicate that Hspalb KO mice are more susceptible to MPTP toxicity. Therefore, reductions in the Hspalb gene level due to various factors, including ageing and genetic defects, result in hightened sensitivity to toxic insults such as MPTP, which affect the pathogenesis of PD. Although it is controversial whether Hsp70 is able to help in the treatment of PD, it is clear that this gene is related to the pathogenesis of PD. We consider that therapeutic methods controlling the level or improving the function of the Hspalb gene may be able to help in the prevention of progression of PD. However, this reuires further study in order to identify the specific molecular mechanisms involved in the relationship between Hspalb and PD.

\section{Acknowledgements}

This work was supported by the Priority Research Centers Program through the National Research Foundation of Korea (NRF) funded by the Ministry of Education, Science and Technology (grant \# 2009-0093829).

\section{References}

1. Wood-Kaczmar A, Gandhi S and Wood NW: Understanding the molecular causes of Parkinson's disease. Trends Mol Med 12: $521-528,2006$.

2. Gwinn-Hardy K: Genetics of parkinsonism. Mov Disord 17: 645-656, 2002

3. McNaught KS and Olanow CW: Protein aggregation in the pathogenesis of familial and sporadic Parkinson's disease. Neurobiol Aging 27: 530-545, 2006.

4. Morimoto RI and Santoro MG: Stress-inducible responses and heat shock proteins: new pharmacologic targets for cytoprotection. Nat Biotechnol 16: 833-838, 1998.
5. Frydman J: Folding of newly translated proteins in vivo: the role of molecular chaperones. Annu Rev Biochem 70: 603-647, 2001.

6. Wegele H, Muller L and Buchner J: Hsp70 and Hsp90 - a relay team for protein folding. Rev Physiol Biochem Pharmacol 151: $1-44,2004$.

7. Klucken J, Shin Y, Masliah E, Hyman BT and McLean PJ: Hsp70 reduces alpha-synuclein aggregation and toxicity. J Biol Chem 279: 25497-25502, 2004.

8. McLean PJ, Klucken J, Shin Y and Hyman BT: Geldanamycin induces Hsp70 and prevents alpha-synuclein aggregation and toxicity in vitro. Biochem Biophys Res Commun 321: 665-669, 2004.

9. Auluck PK, Chan HY, Trojanowski JQ, Lee VM and Bonini NM: Chaperone suppression of alpha-synuclein toxicity in a Drosophila model for Parkinson's disease. Science 295: 865-868, 2002.

10. Dong Z, Wolfer DP, Lipp HP and Bueler H: Hsp70 gene transfer by adeno-associated virus inhibits MPTP-induced nigrostriatal degeneration in the mouse model of Parkinsondisease.Mol Ther 11: 80-88, 2005.

11. Quigney DJ, Gorman AM and Samali A: Heat shock protects PC12 cells against MPP toxicity. Brain Res 993: 133-139, 2003.

12. Wu YR, Wang CK, Chen CM, et al: Analysis of heat-shock protein 70 gene polymorphisms and the risk of Parkinson's disease. Hum Genet 114: 236-241, 2004.

13. Turturici G, Sconzo G and Geraci F: Hsp70 and its molecular role in nervous system diseases. Biochem Res Int 2011: 618127, 2011.

14. Muchowski PJ and Wacker JL: Modulation of neurodegeneration by molecular chaperones. Nat Rev Neurosci 6: 11-22, 2005.

15. Milner CM and Campbell RD: Structure and expression of the three MHC-linked HSP70 genes. Immunogenetics 32: 242-251, 1990.

16. Kim SN, Kim ST, Doo AR, et al: Phosphatidylinositol 3-kinase/ Akt signaling pathway mediates acupuncture-induced dopaminergic neuron protection and motor function improvement in a mouse model of Parkinson's disease. Int J Neurosci 121: 562-569, 2011.

17. Schulz JB and Beal MF: Mitochondrial dysfunction in movement disorders. Curr Opin Neurol 7: 333-339, 1994.

18. Przedborski S, Tieu K, Perier C and Vila M: MPTP as a mitochondrial neurotoxic model of Parkinson's disease. J Bioenerg Biomembr 36: 375-379, 2004.

19. Ryu EJ, Harding HP, Angelastro JM, Vitolo OV, Ron D and Greene LA: Endoplasmic reticulum stress and the unfolded protein response in cellular models of Parkinson's disease. J Neurosci 22: 10690-10698, 2002.

20. Dawson TM and Dawson VL: Molecular pathways of neurodegeneration in Parkinson's disease. Science 302: 819-822, 2003.

21. Beere HM, Wolf BB, Cain K, et al: Heat-shock protein 70 inhibits apoptosis by preventing recruitment of procaspase- 9 to the Apaf-1 apoptosome. Nat Cell Biol 2: 469-475, 2000.

22. Warrick JM, Chan HY, Gray-Board GL, Chai Y, Paulson HL and Bonini NM: Suppression of polyglutamine-mediated neurodegeneration in Drosophila by the molecular chaperone HSP70. Nat Genet 23: 425-428, 1999.

23. Shen HY, He JC, Wang Y, Huang QY and Chen JF: Geldanamycin induces heat shock protein 70 and protects against MPTPinduced dopaminergic neurotoxicity in mice. J Biol Chem 280: 39962-39969, 2005.

24. Nagel F, Falkenburger BH, Tonges L, et al: Tat-Hsp70 protects dopaminergic neurons in midbrain cultures and in the substantia nigra in models of Parkinson's disease. J Neurochem 105: 853-864, 2008.

25. Gao L, Diaz-Martin J, Dillmann WH and Lopez-Barneo J: Heat shock protein $70 \mathrm{kDa}$ over-expression and 1-methyl4-phenyl-1,2,3,6-tetrahydropyridine-induced nigrostriatal degeneration in mice. Neuroscience 193: 323-329, 2011.

26. Shimshek DR, Mueller M, Wiessner C, Schweizer T and van der Putten PH: The HSP70 molecular chaperone is not beneficial in a mouse model of alpha-synucleinopathy. PLoS One 5: e10014, 2010.

27. Meriin AB and Sherman MY: Role of molecular chaperones in neurodegenerative disorders. Int J Hyperthermia 21: 403-419, 2005. 